\title{
A rare case of medulloblastoma with excessive nodularity: imagistic features
}

\author{
A. Tascu, R.E. Rizea \\ 1-st Neurosurgical Department, Hospital Bagdasar-Arseni, Bucharest \\ Neurosurgical Department, U.M.F. Carol Davila, Bucharest
}

\begin{abstract}
Medulloblastoma is the most common malignant tumor of childhood. Neuroimaging can play a role in the diagnosis of medulloblastoma, however atypical features do exist [2]. We report the case of a 1 year and 10- month-old infant diagnosed with a medulloblastoma with what we term "excessive" nodularity based on neuroimaging features and confirmed by neuropathology. CT-scan (CT) and magnetic resonance imaging (MRI) examination of the brain revealed a very large posterior fossa tumor attached to tentorium. On T2-weighted and post-gadolinium sequences, the tumor shows an extensive nodular grape-like appearance. Initial the patient was underwent a ventriculoperitoneal shunt. The second operative procedure was tumour resection. Histology examen revealed a diagnosis of medulloblastoma desmoplastic with extensive nodularity. The neuroradiographic features of this medulloblastoma with what we describe as "excessive" nodularity are important to recognize as these children may be cured with chemotherapy alone.
\end{abstract}

Key words: childhood brain tumor, medulloblastoma, "excessive" nodularity.

\section{Introduction}

In United States primary childhood central nervous system brain tumors occur at an incidence of 5.0 cases per 100,000/personyears. Medulloblastoma is the most common malignant tumor of childhood and represents $13 \%$ of all childhood primary brain tumors ages $0-14$ years [1]. Medulloblastoma is a solid, homogenously enhancing, midline tumor of the posterior fossa. As a primitive neuroectodermal tumor (PNET), it predominantly affects the cerebellum and IV ventricle. Its unique clinico-radiological pattern is referred as "typical" medulloblastoma. However, such typical subset of the disease is not common in clinical practice. Neuroimaging can play a role in the diagnosis of medulloblastoma, however atypical features do exist [2]. We report the case of a 1 year and 10 - month-old infant diagnosed with a medulloblastoma with what we term "excessive" nodularity based on neuroimaging features and confirmed by neuropathology. The specific neuroradiographic features of this 
medulloblastoma subtype are important to recognize as these patients have improved survival and may warrant the correct therapy $[3,4]$.

\section{Case Report}

A 1 year and 10-month-old full term girl presented to our hospital following a 2 weaks history of delayed motor milestones with headache, vomiting, vertigo, ataxia and enlarging head circumference. Her neurological examination revealed moderate axial hypotonia without obvious dysmetria. CT-scan (CT) (figure 2) and magnetic resonance imaging (MRI) examination of the brain revealed a very large posterior fossa tumor attached to tentorium. On T2-weighted and post-gadolinium sequences, the tumor shows an extensive nodular grape-like appearance (figure 1). MRI of the spine was negative for leptomeningeal spread (not shown). Given the patient's young age and unique neuroimaging characteristics the patient is proposed to surgery. Initial the patient was underwent a ventriculo-peritoneal shunt. The second operative procedure was tumour resection. The tumour was fleshy, very vascular and had no direct attachment to the dura. Intraoperative histology examen revealed a diagnosis of medulloblastoma desmoplastic with extensive nodularity. The nodular areas have an impressive neuroradiographic correlate on both T2 and postgadolinium T1-weighted sequences. After surgery the patient had numerous small areas of residual tumour that were scattered throughout the cerebellum (control CT scan figure 3). Given to histology and improved neurological examination after operative procedure, it was decided to proceed with chemotherapy and the oncological treatment is begin.

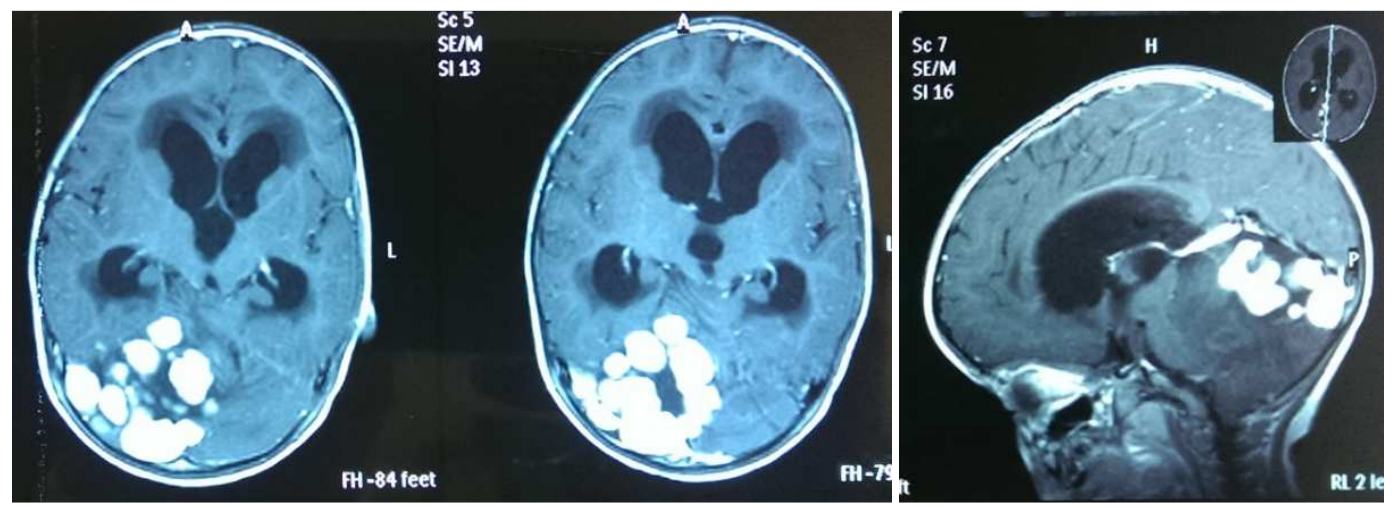


DOI: 10.2478/romneu-2014-0041

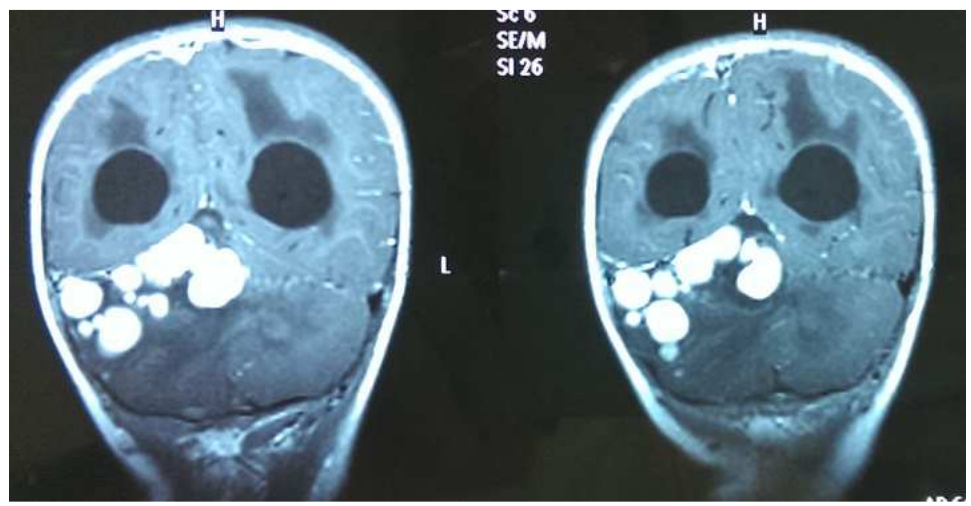

Figure 1 - Preoperative MRI T2 post-gadolinium

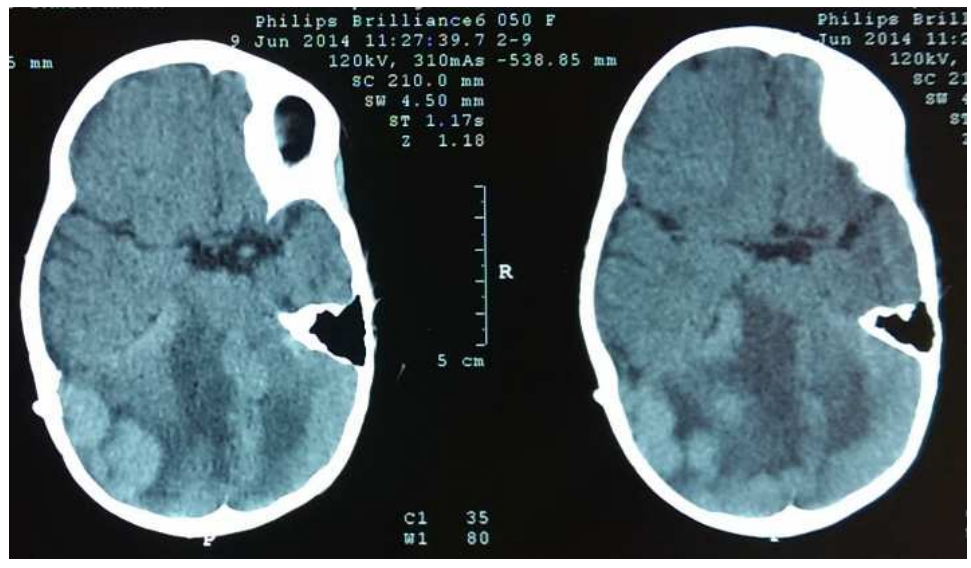

Figure 2 - Preoperative CT-Scan

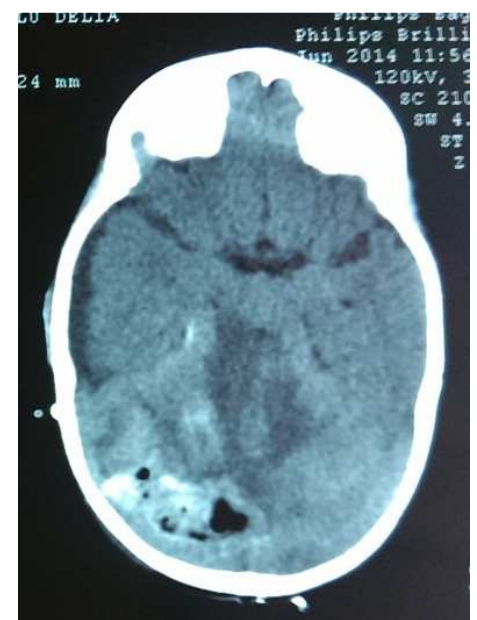

Figure 3 - Postoperative CT-scan

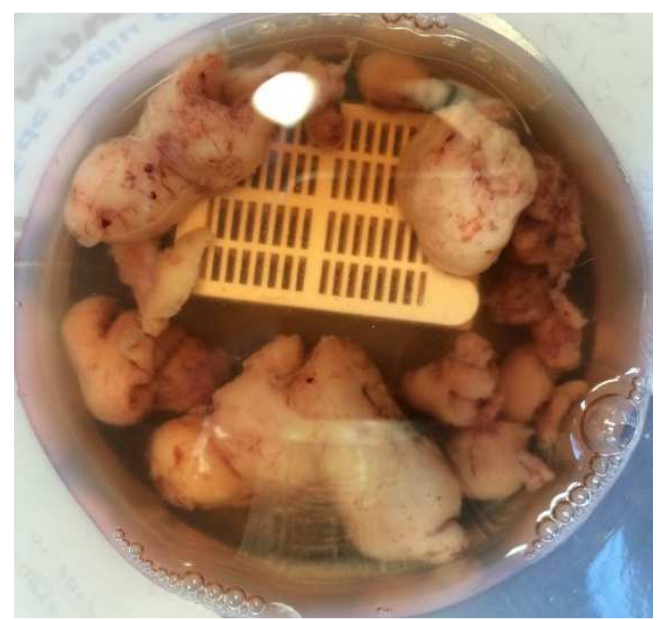

Figure 4 - Extensive Nodularity - aspect of tumour after excision 


\section{Discussion}

Medulloblastoma is a primitive neuroectodermal tumor arising in the posterior fossa. It most commonly affects children with three fourths of patients being under nine years of age. It accounts for $20-25 \%$ of all pediatric brain tumors [5]. Cell of origin of medulloblastoma is still controversial. It has been speculated to arise from cells of external granular layer of cerebellum or from undifferentiated cells of posterior medullary velum. Location of the tumor within the posterior fossa depends on the age of the patient. These are predominantly located in the midline vermis in children. Adults are likely to have heterogeneous cerebellar hemisphere tumors, which is due to a greater prevalence of desmoplastic variant. Medulloblastomas are known to spread by CSF pathways. Gupta et al has reported a case of multicentric medulloblastoma where the metastatic nodules were located in the third ventricle [6].

The five-year overall survival of children with average risk medulloblastoma who are treated with a combination of craniospinal radiation and chemotherapy approaches $90 \%$. The newly stratified molecular classification of medulloblastoma has revealed distinct subsets of children who have better prognosis in spite of their young age. Among these children are those with either nodular desmoplastic or extensive nodularity. The neuroradiographic features of this medulloblastoma with what we describe as "excessive" nodularity (fig 4) are important to recognize as these children may be cured with chemotherapy alone and spared from the neurocognitive consequences of radiation therapy. Similar finding of medulloblastoma with "extreme" nodularity has been reported [7]. The differential diagnosis of similar grape-like characteristics of a posterior fossa mass in a patient of similar age would include atypical teratoid rhabdoid tumor, atypical choroid plexus carcinoma, and dysplastic gangliocytoma of the cerebellum. The distinguishing feature between dysplastic gangliocytoma and medulloblastoma with extensive nodularity is the avidity for gadolinium enhancement in the latter. Our case study significantly enhances our understanding of the neuroradiographic features of this uncommon tumor.

\section{Correspondence}

Assoc. Prof. A. Tascu

e-mail:tascu_alexandru@yahoo.com

\section{References}

1. CBTRUS, "Primary brain and central nervous system tumors diagnosed in the United States in 2004-2008," CBTRUS Statistical Report, Central Brain Tumor Registry of the United States, Hindsdale, Ill, USA, 2012.

2. A. Eran, A. Ozturk, N. Aygun, and I. Izbudak, "Medulloblastoma: atypical CT and MRI findings in children," Pediatric Radiology, vol. 40, no. 7, pp. 1254$1262,2010$.

3. M. Massimino, M. Antonelli, L. Gandola et al., "Histological variants of medulloblastoma are the most powerful clinical prognostic indicators," Pediatric Blood $\&$ Cancer. In press.

4. F. Giangaspero, G. Perilongo, M. P. Fondelli et al., "Medulloblastoma with extensive nodularity: a variant with favorable prognosis," Journal of Neurosurgery, vol. 91, no. 6, pp. 971-977, 1999.

5. Mueller DP, Moore SA, Sato Y, Yuh WT. MRI spectrum of medulloblastoma. Clin Imaging 1992;16:250-5.

6. Prasad VS, Reddy DR, Sundaram C. Calcified medulloblastoma in a child. Clin Imaging 1994;18:275-6. 7. G. Arora, A. Garg, G. Singh et al., "Medulloblastoma with extreme nodularity," Neurology India, vol. 59, pp. 634-635, 2011. 